\title{
Neuro-Fuzzy for Sensor Fault Detection and Isolation
}

\author{
Rajendra Sharma
}

Snehal Kokil

Priti Khaire

\begin{abstract}
This paper presents the sensor fault configuration through Neuro-Fuzzy. As we know that sensor faults have been observed in may domain. Various sensors faults are present such as bias, scaling, drift so to remove this kind of fault which is present we make the sensor to reconfigure to normal condition and this reconfiguration is done through NeuroFuzzy which uses the expert knowledge stored in them while training. This technique is implemented through ANFIS tool. Sugeno-Type fuzzy inference system is used, which is adaptive in nature and also Gaussian membership function is used. This technique uses the hybrid optimization which consists of combination of backpropagation and least square method algorithm. Simulation result is shown.
\end{abstract}

\section{Keywords}

Neuro-Fuzzy, Expert system, Sensor fault tolerance, Fault detection and isolation in sensors

\section{INTRODUCTION}

Sensors play a vital role in modern application systems to utilize their full benefits. In some application system sensors are correlated with the number of other sensors used in various systems. Important concepts like uptime also authoritative command continuous state analysis with sensors. However application system has attained the fame as being the "weak link". Sensor failure has been observed for highly complex system. Sensor failure result into system breakdowns such as aborted take-off of the space shuttle. Sensor failure in aerospace domain has been observed from many decades. The literature is full of example where a sensor malfunction led to either loss of mission or life.

- The sensor failures can also lead to fatal accident of B-12 bomber aircraft on $23^{\text {th }}$ February, 2008. The accident was because of sensor which distorted three of the aircrafts twenty-four sensor reading forcing the bomber to pitch up on takeoff, it result into stalling and subsequent crash. The sensor reading confused the pitch and result in the loss of this $\$ 1.4$ billion in term of money.

- The Brazil-to-France flight plunged into the Atlantic Ocean on $1^{\text {st }}$ June, 2009 killing all 288 people on board. The investigation report show that pilot failed to respond effectively problems with the plane's speed sensor and to correct its trajectory when things first started to go wrong.

- The Bureau of investigating and analysis told the data indicated that flight 447 crashed because of the aircraft's speed sensor gave invalid reading.

To deal with these kind of issue through multiple redundancy often come with weight, cost and complexity penalties and not guaranteed to improve problem. In the last few decades, mathematical model-based fault diagnosis technique has received a great deal of investigation. This is due to increasing complexity of modern engineering system and economics factor detection, isolation and estimation for sensor system in presence of uncertainties is still a challenging task. Researchers have explored the fault diagnosis for sensor system using knowledge based methodologies where they use different online approximation techniques to estimate the deviation of system caused the fault. Sensor fault detection and isolation can be implemented through Neuro-fuzzy system by using the hybrid optimization algorithm.

\section{ISSUES IN SATELLITE SENSORS}

There are mainly some of faults which occurring in aerospace domain for sensors. In literature some sensor fault has been provided and they are categories are as follows:

- Bias: It occurs due to incorrect physical changes in the sensor system. The equation is $Y_{f}=X+\beta+$ noise, where $\beta$ constant offset value is. As a result time variation may produce drift failure.

- Drift: Normal statistics of the sensor signal may vary due to time varying offset. In literature nonlinear drift is also possible. The equation of failure drift is $Y_{s}=X+\delta(t)+$ noise, where time varying offset is $\delta(t)$.

- Scaling: This problem may arise due to noise, hard fault or intermittent. In this magnitudes are scaled by a factor $\alpha(t)$. the equation of scaling problem is $Y_{f}=\alpha(t)+$ $X+$ noise, where $0<\alpha(t)<\infty$ Time varying may be scaling constant

\section{LITERATURE SURVEY}

There are various approaches have been implemented for sensor fault detection and isolation. In literature hardware redundancy approaches are used in high certainty in detection and isolation of faulty sensor with easy implementation. However the use of redundant sensor may not always be feasible due to cost and space constraint. It might be possible that redundant sensor may fail with similar symptoms because they work in the same environment. [Patton et.al, 1989]. In [Broen, 1974], a class of voter estimates, derived from leastsquare estimation, was developed to detect and isolate a family sensor. Inspired by the same idea, the method in [Desai and Ray, 1989] concurrently checked the relative consistencies among all redundant measurement, and utilities most consistent and inconsistent subset for the estimation of measured variable and detection of a faulty sensor. In order to overcome the limitation of a static threshold, the concept of fuzzy sets was used to [park and Zee, 1993] to develop a rule -based diagnostic logic for a set of redundant sensors so that the dynamic disturbance and noise in the measurement can be taken into account Knowledge-based expert system. In [Chandrasekaran and Punch, 1988], an approach based on hierarchical classification was developed for the sensor validation by adding the associated information into the knowledge group predicted the so. Although such an expert system can detect a faulty sensor or any other faulty component in the same way the model based reasoning approach, which is specific to the application domain. In [Betta et.al, 1995], knowledge based analytical redundancy approach was proposed to integrate both qualitative models 
and empirical knowledge into an expert system. In order to implement such an expert system to a measurement station consists a large number of sensors with high uncertainty, the algorithm was developed in [Betta et.al, 1997]. Despite its capability its capability in dealing with qualitative knowledge, the performance of the expert system depends mainly in its design ranging from the different ways of embedding the existing knowledge to the selection of fault threshold. Moreover an expert system requires great effort during for the development, but it has certain limitation capability in handling dynamic system [Hotclling, 1933], [Pearson, 1901]. PCA (Principal Component Analysis) method has been used to compress a large amount of correlated data into a lowdimension data set. There is a limitation for linear transformation for use of PCA, method for a nonlinear process lead which to the loss of important information [Xu.et.al, 1992]. To over such drawback, a generalized PCA was proposed [Gnandesikan, 1997]. The use of PCA for sensor validation was also applied in [Kerchen et.al, 2005] for the monitoring of structure health. In [Benitez-Perez et.al, 2005], a self-organizing map (SOM) was first trained for the normal and various faulty process behavior based on the PCA. Faulty isolation was then used by calculating the similarity between the observations with the trained SDM. In [Ch. Et.al, 2004], a sensor fault detection method was proposed using Kernel. PCA based on two statistics that were defined as the contribution of each variable to the monitoring statistic. A Bayesian network is a directed acyclic graph which contains a set of random variable and its probabilistic dependencies. It has a finite set of mutually exclusive state; node is connected with a causal relationship. Each child node is associated with a parent node and priori probability is associated with root node. Probabilistic inference procedure is updated in Bayesian network through probability distribution, when new observation is available for evidence. Bayesian network is represented with a graph, $\mathrm{G}=(\mathrm{V}, \mathrm{E})$, it contains the node $\mathrm{X}=$ $(\mathrm{Xv}) \mathrm{v} \in \mathrm{V}, \mathrm{V}=(1,2,3,4,5)$, having a set of random variables and directed link. $\mathrm{E}=\{(\mathrm{X} 1, \mathrm{X} 2),(\mathrm{X} 1, \mathrm{X} 4),(\mathrm{X} 2, \mathrm{X} 4),(\mathrm{X} 4$, $\mathrm{X} 5)\}$ Which shows the casual relationships among variables $(\mathrm{X} 1, \mathrm{X} 2)$ represents the root variable and probabilities associated with directed link as E, The probability of any linked distribution can be computed using chain rule, $\mathrm{P}(\mathrm{X} 1=\mathrm{x} 1 \ldots \mathrm{Xn}=\mathrm{xn}) \quad=\prod_{v=1}^{n} P(X v=X v \mid X v+1=X v+1=$ $X v+1 \ldots X n=X n)$. The Bayesian networks were used to solve the sensor faults for detection and identification. [RojarGuzman and Kramer, 1993, Ibarguengoytia 2001, 2006, Mehranbod et. al., 2008, Krishnamoorthy, 2010]. Sensor validation play a vital role for this [Nicholson and Brady, 1994, Aradhye, 2002, Mehranbod et. at., 2005] various methods were proposed used in dynamic Bayesian for this importance of sensor validation during process transition. In recent years various new technique for sensor fault detection and isolation had been implemented such Kalman Filter and Knowledge based approach [Jonny Carlos da Silva, Abhinav Saxena, Edward Balaban, Kai Goebel (2012)]

\section{DESCRIPTION OF TESTBED}

The testbed which is demonstrated in Balaban et.al (2009) are used for research. Initial experiment a ball screw electromechanically actuator was used as plant and experiment were performed on moog. In this experiment actuator were connected to hydraulic load cylinder through a rotating horn. The dSPACE platform was used for control and data acquisition which is real-time software. Test actuator, vibration was measured at four points. Vibration was measured at z-direction using an accelerometer which is mounted direct on the nut and ball screw.

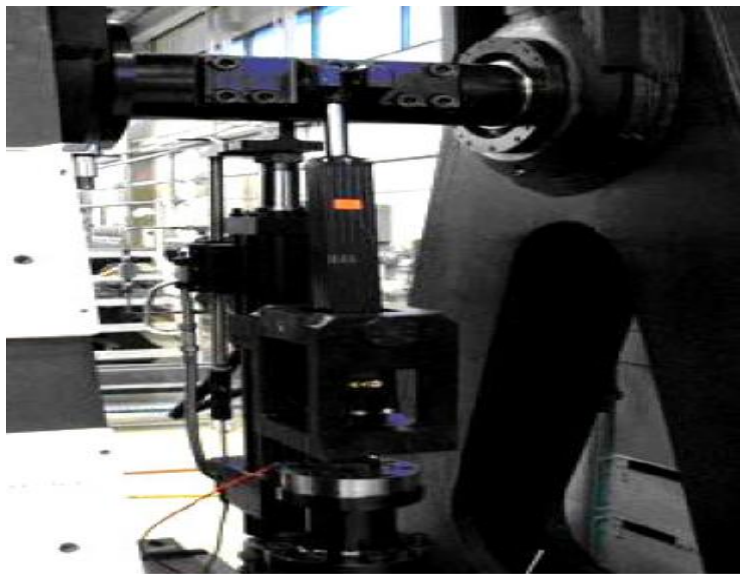

Fig 1: Testbed

For sensing the temperature, T-type thermocouple sensor was mounted on the nut and a resistance temperature was used in the stator of the motor. Load cell is used to sense load. Linear differential voltage transducer was used for the position of the rod end of the test. The motor drive output an analog signal representing the torque producing current as well as motor velocity for data acquisition. Sensor fault were injected a posteriori. For each of the mechanical component fault, permutations of the following conditions were used to run $2 \times 2 \times 2=8$ scenarios.

- Motion profile: Triangular or sinusoid wave.

- Load type: Spring or constant.

- Load level: High (1725 lbs. spring force, $1800 \mathrm{lbs}$. constant force) and low (860 lbs. spring force, 900 lbs. constant force)

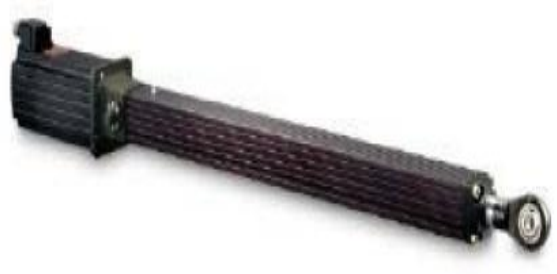

Fig 2: Moog MaxForce 883-023 test actuator

Since the above experiments produced short segments of data at fixed operating condition, so splicing data from different types of experiments. The new scenario was design to preserve, the newly data collected as much as possible, while extending the duration of 180 s each and varying load and position profiles. These scenarios contain two main part the initial part consists of normal data and later part consist of fault data (90s per segment). Since the hardware limitations of the test stand wanted that then faults be seeded before the corresponding experiments began, normal data taken from experiments conducted under the same condition. Mechanical fault were observed in EMA systems. In the experimental data to generate scenario with sensor data fault were simulated. To distinguish between the sensor and system fault, diagnostic classifier were used. Specific sensor fault types were selected based on the type of sensors. .used in the testbed and their common fault modes available from the literature. 


\section{IMPLEMENTATION THROUGH ANFIS TOOLBOX}

The low load condition data were taken the normal data and faults present data are considered. Both this data are merged so that if there is variation present in data can be identified. Initial normal 500 sensor reading and fault 500 sensor reading is taken and performed the time series analysis, so that if there is an deflection is present than it can be identified. This operation is carried on four different sensors such as Motor X Temperature, Motor X Nut Temperature, Motor Y Nut Temperature and Motor Y Current while performing the time series analysis we can classify the normal data and faulty data. In this Gaussian member function are used and linguistic variables used for input and output are low, medium and high. Rules are constructed are as follows, and these rules can be viewed in as followed:

- If sensor reading is low then sensor output is low.

- If sensor reading is medium then output is medium.

- If sensor reading is high then output is high.

Training data set is loaded with faulty and correct sensor reading Target value are taken for normal sensor is same as normal and faulty as mean value of sensor reading so that they lie in same normal reading range and initial fuzzy inference system structure is generated. The set of normal sensor and faulty sensor reading are passed to Sugeno type system for training. While performing the training Gaussian membership function are used and epoch is 20 is taken and training is carried out using hybrid optimization which is consists of combination of Back propagation and Least square method. When fault is detected after that faulty sensor is reconfigured to the normal condition, this technique is implemented. Target value are taken for normal sensor is same as normal and faulty as mean value of sensor reading so that they lie in same normal reading range. The set of normal sensor and faulty sensor reading are passed to Sugeno type system for training. After training then testing of data is performed so that model can be validated. A set of faulty data is taken and check whether it is operating the correct reconfiguration of faulty sensor reading. If it is performing the correct output then model is valid and simulation shows that faulty sensor reading are correctly configured to normal condition.

\section{RESULT AND DISCUSSION}

\subsection{Sensor 1: Motor X Temperature}

As we can see that normal data range of sensor had been varied to the faulty data range, so it important to reconfigured to the normal condition. From the figure we see can that Neuro-Fuzzy have reconfigured to the normal condition. Compare of normal, faulty and corrected sensor reading done in Figure 3.Other sensor value is configured, Motor X Nut Temperature, Motor Y Nut Temperature and Motor Y Current is shown in figure 4, 5, 6 and 7.

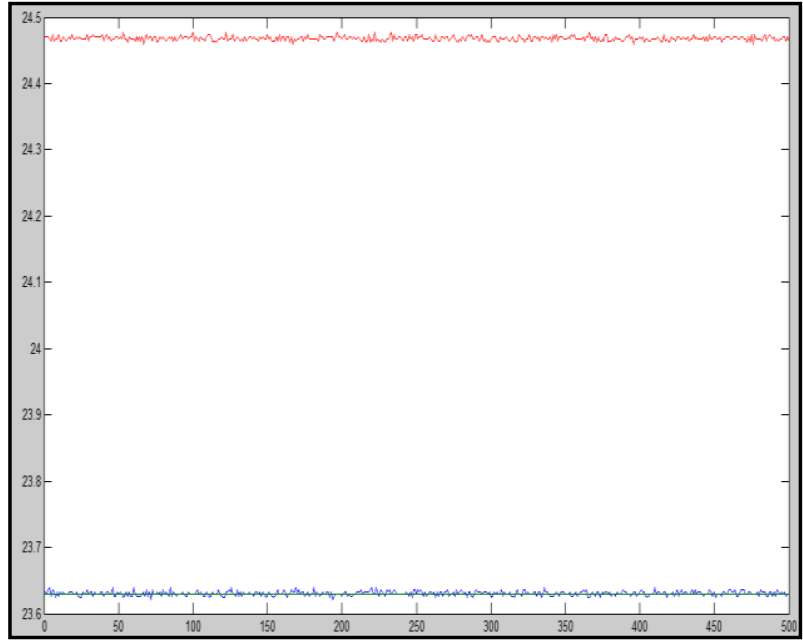

Fig 3: Comparing normal, faulty and correct sensor reading

\subsection{Sensor 2: Motor X Nut Temperature}

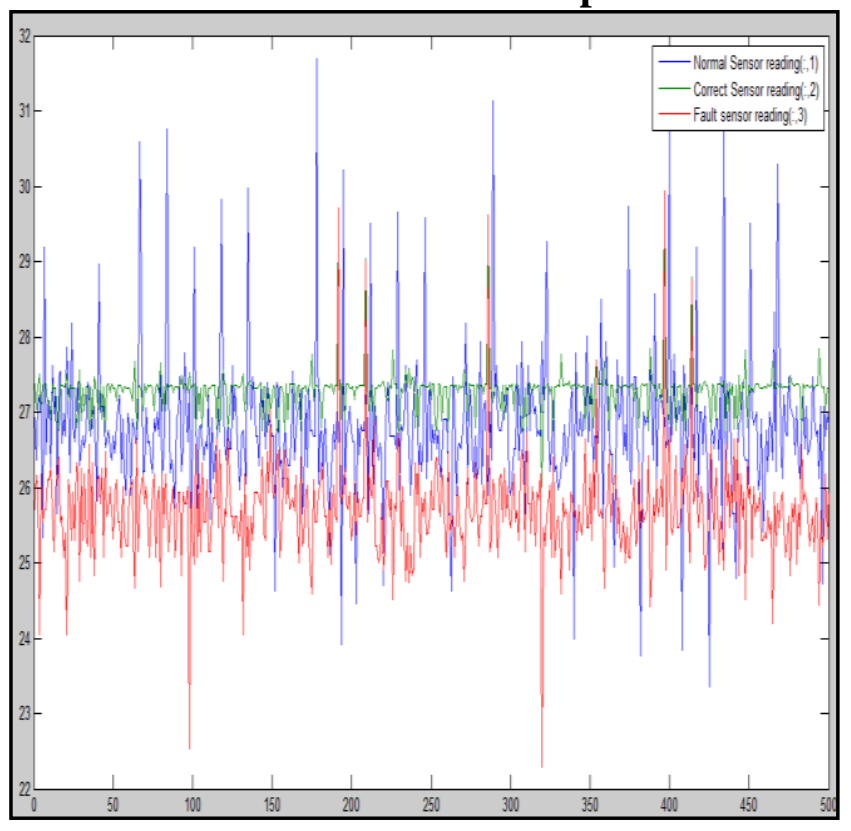

Fig 4: Comparing normal, faulty and corrected sensor reading 


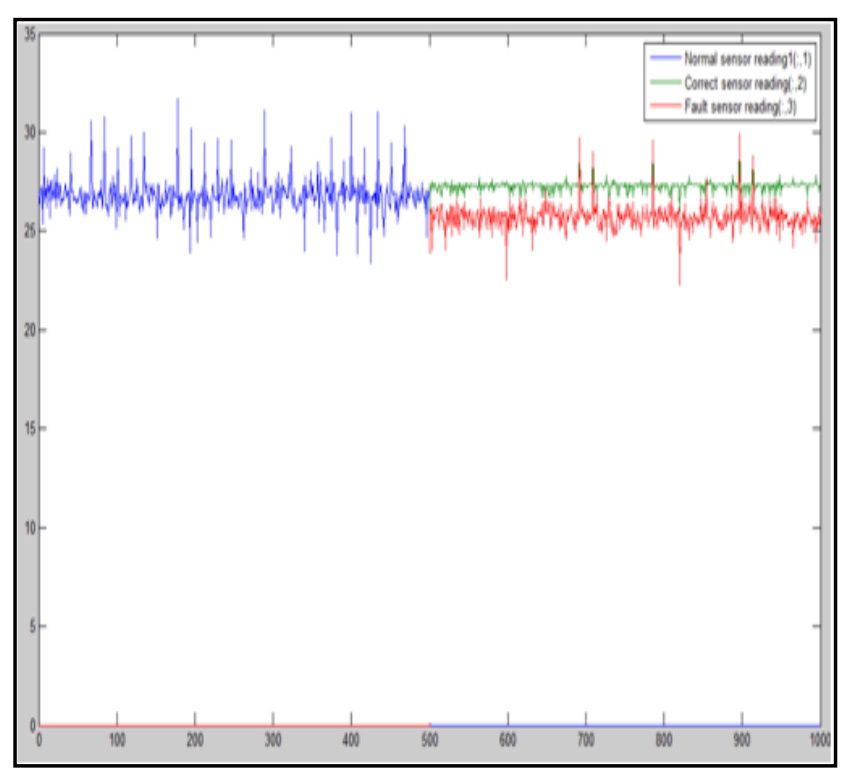

Figure 5: Comparing normal, faulty and corrected with continuous time series analysis.

\subsection{Sensor 3: Motor Y Nut Temperature}

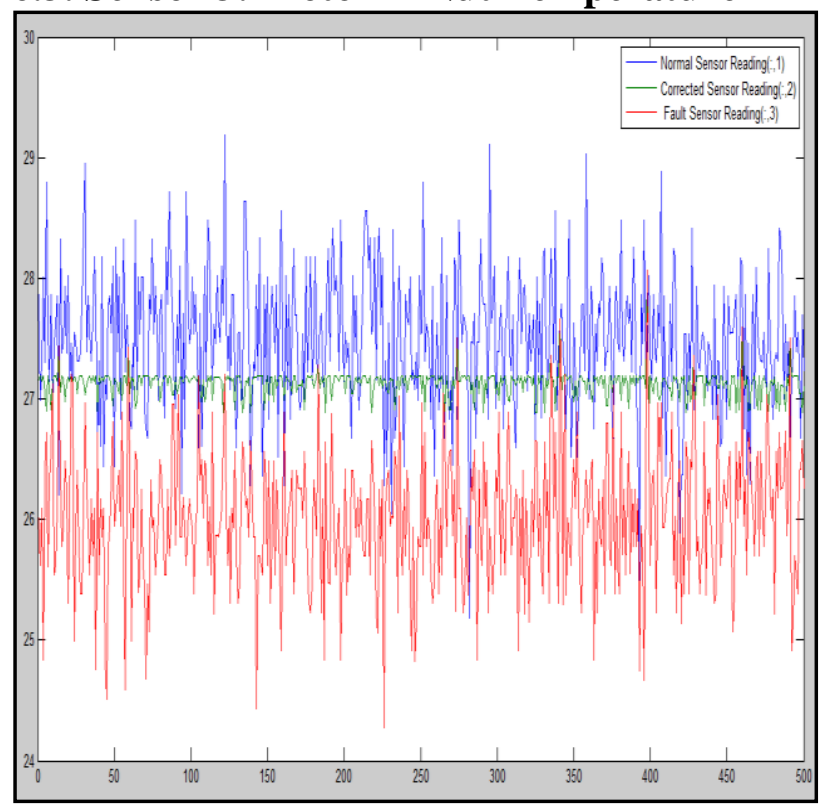

Fig 6: Comparing normal and faulty and corrected sensor reading

\subsection{Sensor 4: Motor Y Current}

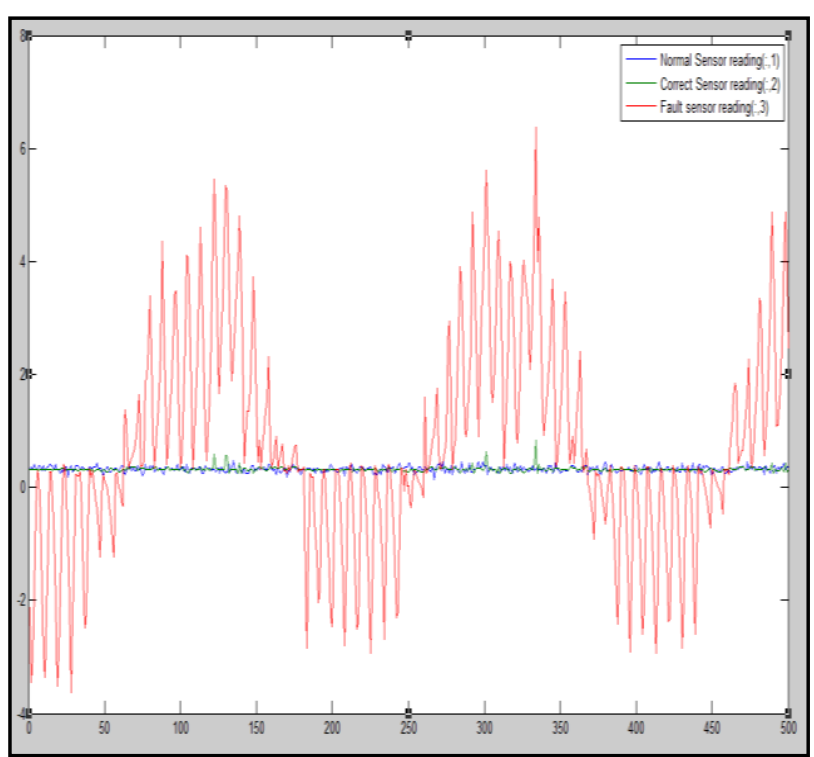

Figure 7: Comparing with normal, faulty and corrected sensor reading

From the above figure we can say that correction of faulty sensor has reconfiguration to normal sensor reading and faulty had been isolated through Neuro-Fuzzy.

\section{CONCLUSION}

This paper is based on Neuro-Fuzzy for sensor fault detection and isolation. To detect the fault which is present in sensor through time series analysis method after identifying the fault such as bias, drift and scaling reading are corrected by reconfiguring to normal condition. Simulated result shows that sensor faults are reconfigured to the normal condition. After the reconfiguration of sensor, use can say that reliability of sensor which results into robust application. This reconfiguration is done using the hybrid optimization technique. To isolate the faults Sugeno-type FIS is used above simulated result is shown. This technique can be implemented in any domain for sensor fault detection and isolation.

\section{FUTURE SCOPE}

There are various domain were sensors are used widely. So a fault occurring in sensor might be large in various complex systems. There are number of faults associated with the real time system such as electrical systems supporting sensor operation, data acquisition (signal conditioning and analogto- digital converter) and there are many other reasons such as intermittencies need to be taken into consideration so that system becomes reliable other works, such as nonlinear drift will be carry out to make the system reliable and robust for other application.

\section{REFERENCES}

[1] Girishkumar sago(2008),"Pilot in Loop Assessment of Fault Tolerance Flight Control System",p1

[2] L. Fortuna, G. Rizzotta, M. Lavorgna, G. Nunnari, M. G. xibilia and R.Caponetto (2011),"Soft Computing",Springer, p.167-173.

[3] Luger, George; Stubblefield, William A. (2004) "Artificial Intelligence: Structures and Strategies for 
Complex Problem Solving ", The Benjamin/Cummings Publishing Company, p227-231

[4] Manish Mahajan, RajdevTiwari (2010), "Introduction to Soft Computing”, Acme Learning Pvt. Ltd., p.9-13, 1719,165-172.

[5] McCorduck, Pamela (2004) "Machines Who Think", Natick, MA: A. K. Peters, Ltd., p. 327-335, 434-435.

[6] Nilsson, Nils (1998) "Artificial Intelligence: A New Synthesis”, Morgan Kaufmann Publishers.p174

[7] R.A Edmunds. (1988) "The Prentice Hall Guide to Expert Systems", Prentice Hall, Englewood,p.130

[8] Robert J.Schalkoff (2011), “Artificial Neural Networks", TATA McGRAW-HILL, p-360-380.

[9] Russell, Stuart J.; Norvig, Peter (2003), "Artificial Intelligence: A Modern Approach", Upper Saddle River, New Jersey: Prentice Hall, p(22-24)

[10] S. N. Sivanandam, M. Paulraj (2011), "Introduction to artificial neural networks", Vikas Publication House Pvt. Ltd., p.8-9.

[11] Li Jiang (2011). Sensor fault detection and isolation using system dynamics. $\mathrm{PhD}$ thesis, University of Michigan

[12] Patton, R. and Chen, J. (1997). Observer-based fault detection and isolation: robustness and applications. Control Engineering Practice, 5(5):671 $\{82$.

[13] Mengshoel, O., Darwiche, A., and Uckun, S. (2008). Sensor validation using Bayesian networks. In the 9th International Symposium on Artificial Intelligence, Robotics and Automation in Space, Los Angelo's, CA.

[14] Park, S. and Lee, C. (1993). Fusion-based sensor fault detection. In Proceedings. IEEE International
Symposium on Intelligent Control (Cat. No.93CH32789), pages 156\{61, Chicago, IL, USA. IEEE.

[15] Pearson, K. (1901). Lines and planes of closest fit. Philosophical Magazine, 2:559\{572.

[16] Xu, L., Oja, E., and Suen, C. (1992). Modified hebbian learning for curve and surface fitting. Neural Networks, $5(3): 441\{57$.

[17] Rojas-Guzman, C. and Kramer, M. (1993). Comparison of belief networks and rule-based expert systems for fault diagnosis of chemical processes. Engineering Applications of Artificial Intelligence, 6(3):191\{202.

[18] Mengshoel, O., Darwiche, A., and Uckun, S. (2008) Sensor validation using Bayesian Networks. In the 9th International Symposium on Artificial Intelligence, Robotics and Automation in Space, Los Angelo's, CA

[19] http://edition.cnn.com/2012/07/05/world/europe/franceair-crash-report

[20] http://www.globalspec.com/reference/

[21] http://info.iet.unipi.it/ lazzerini/icse/FLToolbox_Estratt o2.pdf

[22] http://www.macrosensors.com/lvdt_tutorial.html

[23] http://www.mycockpit.org/forums/content/378-forcesensors.html

[24] http://www.ni.com/cms/images/devzone/tut/c/cb971a32 991.gif

[25] http://nktechnologies.com/current-sensing.html

[26] http://nktechnologies.com/current-sensing.html

[27] http://www.omega.com/techref/ 